\title{
PREVALÊNCIA DE INFECÇÃO PELO VÍRUS DA HEPATITE C EM PACIENTES COM DIABETES MELITO TIPO 2
}

\author{
Mônica Beatriz PAROLIN ${ }^{1}$, Rosângela RÉA ${ }^{2}$, Rosa Maria VARGAS², \\ Ana Cristina Ravazzani de ALMEIDA ${ }^{2}$, Giorgio Roberto BALDANZI ${ }^{3}$ e Reginaldo Werneck LOPES $^{4}$
}

RESUMO - Racional - Estudos recentes têm sugerido possível associação entre infecção pelo vírus da hepatite C (VHC) e diabetes melito tipo 2, relatando prevalência elevada de infecção pelo vírus C nessa população. Objetivo - Verificar a prevalência de infecção pelo VHC em adultos portadores de diabetes melito em nosso meio. Métodos - Foram avaliados consecutivamente 145 adultos com diabetes melito tipo 2 e 104 com diabetes melito tipo 1, em acompanhamento no ambulatório de diabetes de um hospital universitário, quanto à presença de anticorpos contra VHC (anti-VHC) por método ELISA de quarta geração, utilizando como grupo controle 16720 doadores de sangue da cidade de Curitiba, PR, no período em que foi realizada a pesquisa. Os pacientes diabéticos foram também avaliados quanto a dados demográficos, clínicos, bioquímicos (níveis séricos de aminotransferases) e exposição prévia a fatores de risco para infecção pelo VHC. Resultados - Maior prevalência de positividade do anti-VHC foi observada em pacientes com diabetes melito tipo 2, em comparação aos doadores de sangue. A prevalência de anti-VHC naqueles com diabete melito tipo 2 foi superior à encontrada no tipo 1 , embora não tenha alcançado significância estatística. Nos dois grupos de diabéticos houve predomínio do sexo feminino, sendo que os do tipo 2 apresentavam idade média superior aos do tipo 1. A distribuição de cor, tempo de diagnóstico do diabetes e a freqüência de exposição a fatores de risco foram semelhantes nos dois grupos. A mediana da taxa de alanina aminotransferase dos pacientes com diabetes tipo 2 foi superior à observada nos do tipo 1. Conclusões - Encontrou-se maior prevalência de infecção pelo $\mathrm{VHC}$ em diabéticos tipo 2 se comparado à população saudável de Curitiba, corroborando publicações prévias em outras populações.

DESCRITORES - Hepatite C. Diabetes mellitus tipo II.

\section{INTRODUÇÃO}

O vírus da hepatite $\mathrm{C}(\mathrm{VHC})$ é uma das principais causas de doença hepática crônica em todo o mundo e a indicação mais freqüente de transplante hepático na atualidade. Estimase que existam hoje, pelo menos, 170 milhões de portadores do VHC e que parte destes irá desenvolver complicações como cirrose e hepatocarcinoma. A infecção pelo VHC tem sido implicada na gênese de algumas doenças extra-hepáticas como a crioglobulinemia mista essencial, porfiria cutânea tardia esporádica, doença da tireóide e glomerulonefrite ${ }^{(8)}$.

Mais recentemente, estudos epidemiológicos sugerem que o diabetes melito (DM) possa representar também manifestação da infecção crônica pelo $\mathrm{VHC}^{(12,13)}$. Vários autores relataram prevalência aumentada de infecção pelo VHC em diabéticos tipo 2, em contraste com a população geral $^{(5,15,18,19)}$. A possível associação entre VHC e DM também é sugerida por estudos transversais que encontraram prevalência aumentada de DM tipo 2 em pacientes com hepatite crônica $\mathrm{C}$, quando comparada à observada em pacientes com outras formas de doença hepática crônica, mesmo na ausência de cirrose $e^{(1,2,6,9,11,13)}$.

Embora sua patogênese não esteja definitivamente estabelecida, o encontro de índices aumentados de resistência insulínica em pacientes transplantados hepáticos portadores de VHC pode representar a base patogênica da associação VHC e DM tipo $2^{(3)}$.

Com o propósito de verificar esta questão em nossa população, que apresenta perfil genético e fatores ambientais próprios, desenvolveu-se estudo transversal para determinar a prevalência de infecção pelo VHC em adultos diabéticos.

\footnotetext{
${ }^{1}$ Serviço de Transplante Hepático; ${ }^{2}$ Serviço de Endocrinologia e Metabologia; ${ }^{3}$ Serviço de Hemoterapia do Hospital de Clínicas da Universidade Federal do Paraná; ${ }^{4}$ Departamento de Clínica Medica da Faculdade de Medicina da Universidade Federal do Paraná, Curitiba, PR.

Endereço para correspondência: Dra. Mônica Beatriz Parolin - Rua Lamenha Lins, 2280 - 80220-080 - Curitiba, PR. E-mail:mbparolin@hotmail.com
} 


\section{MATERIAL E MÉTODOS}

No período de agosto de 2001 a março de 2002 foram avaliados, consecutivamente, 145 adultos (idade $\geq 18$ anos) com DM tipo 2 e 104 adultos com tipo 1, em acompanhamento regular no Ambulatório de Diabetes do Serviço de Endocrinologia e Metabologia do Hospital das Clínicas da Universidade Federal do Paraná - HCUFPR, Curitiba, PR, sem investigação prévia para hepatite C. Os pacientes incluídos no estudo foram entrevistados e tiveram seus prontuários médicos revisados. Os seguintes dados foram coletados: idade atual, sexo, cor, tempo de diagnóstico do DM e exposição prévia a fatores de risco para infecção pelo VHC.

Os pacientes selecionados submeteram-se a determinação dos valores séricos da alanina aminotransferase (ALT; homens: $10-40 \mathrm{UI} / \mathrm{L}$; mulheres $7-35 \mathrm{UI} / \mathrm{L}$ ) e à pesquisa de anticorpos contra o VHC (anti-VHC) por método de enzimoimunoensaio (ELISA) de quarta geração (ABBOTT Murex Anti-HCV 4.0, Abbott Diagnostic, Chicago, IL, EUA). Todos os resultados positivos foram repetidos, sendo considerados positivos apenas os que persistiram reagentes.

Como grupo controle foram utilizados 16.720 doadores de sangue atendidos no Banco de Sangue do HC-UFPR no período de janeiro a dezembro de 2002. Cabe ressaltar que o grupo controle representado por doadores de sangue não é uma população sabidamente diabética, visto que indivíduos com diagnóstico prévio de diabetes não são habitualmente aceitos para doação de sangue. O teste empregado para detecção anti-VHC foi o mesmo utilizado nos pacientes diabéticos. Todos os doadores com testes reagentes para o anti-VHC tiveram suas amostras repetidas em duplicatas, sendo considerados positivos apenas os que persistiram reagentes. A pesquisa foi aprovada pelo Comitê de Ética e Pesquisa em Seres Humanos do HC-UFPR.

As variáveis categóricas foram expressas em percentagens e as contínuas em média \pm desvio padrão ou mediana (variação), de acordo com sua distribuição. Na comparação do grupo de pacientes com DM tipo 1 e tipo 2 foram empregados os seguintes testes estatísticos: teste $t$ de Student, teste de Mann-Whitney e teste Qui-quadrado de Pearson. Na comparação da prevalência de infecção pelo VHC entre portadores de DM tipo 2 e doadores de sangue empregou-se teste Qui-quadrado de Pearson. Para os testes aplicados adotou-se nível de significância de $5 \%(P<0,05)$.

\section{RESULTADOS}

As principais características dos pacientes diabéticos tipos 1 e 2 avaliados estão dispostas na Tabela 1 . Nos dois grupos houve predomínio de indivíduos de cor branca e do sexo feminino, sendo que diabéticos tipo 2 apresentaram média de idade superior aos do tipo $1(55,25 \pm 11,24$ vs. $24,32 \pm 7,12$; $P<0,01)$. A prevalência de anti-VHC nos diabéticos tipo 2 foi maior que a do grupo tipo $1(2 \%$ vs. $0 \%, P=0,14)$, embora não se tenha alcançado significância estatística, possivelmente pelo tamanho reduzido da amostra. A mediana do nível sérico de ALT dos pacientes com DM tipo 2 foi significativamente maior à encontrada no grupo tipo 1 . Em relação à exposição prévia a fatores de risco para hepatite $\mathrm{C}$ os dados estavam disponíveis nos 104 pacientes com DM tipo 1 e em 142 dos 145 do tipo 2. Houve tendência de maior ocorrência de fator de risco no grupo tipo 2, embora com nível de significância limítrofe.

TABELA 1 - Características de 249 pacientes diabéticos avaliados quanto a prevalência de infecção pelo vírus da hepatite $C$

\begin{tabular}{lccc}
\hline Variáveis & DM tipo $1(\mathbf{n}=104)$ & DM tipo $2(\mathbf{n}=145)$ & Valor P \\
\hline Idade (anos) & $24,32 \pm 7,12$ & $55,25 \pm 11,42$ & $<0,01$ \\
Sexo feminino (\%) & $63(60,57)$ & $96(66,20)$ & 0,36 \\
Raça caucasiana (\%) & $75(72,11)$ & $91(62,75)$ & 0,25 \\
Tempo de DM & $9(0,1-28)$ & $7(0,5-36)$ & 0,08 \\
anos, mediana) & & & \\
Anti-VHC + (\%) & $0(0)$ & $3(2)$ & 0,14 \\
ALT (UI/L) (mediana) & $15(6-159)$ & $18(7-177)$ & $<0,01$ \\
$\begin{array}{l}\text { Fatores de risco para } \\
\text { VHC (\%) }\end{array}$ & $7 / 104(6,73)$ & $20 / 142(14,08)$ & 0,068 \\
\hline
\end{tabular}

$\mathrm{DM}=$ diabetes melito

$\mathrm{VHC}=$ vírus da hepatite $\mathrm{C}$

ALT = alanina aminotransferase

Ao se considerar o grupo total de diabéticos $(n=249)$ e comparar os 3 pacientes anti-VHC $(+)$ com os com 246 anti-VHC (-), não se observou diferença estatisticamente significante em relação à idade média, raça, tempo de evolução do DM, taxas de ALT e exposição a fatores de risco. Quando se analisou apenas o grupo com DM tipo $2(n=145)$ constatouse que os 3 pacientes anti-VHC (+) e os 142 anti-VHC (-) se comportaram de maneira semelhante em relação à idade $(P=0,45)$, distribuição por sexo $(P=0,21)$ e raça $(P=0,61)$, tempo de evolução do $\mathrm{DM}(P=0,65)$ e exposição a fatores de risco $(P=0,33)$. A única variável na qual se observou diferença estatisticamente significante entre os diabéticos tipo 2 anti-VHC (+) e os anti-VHC (-) foi a mediana da taxa sérica de ALT (64 UI/L vs. $18 \mathrm{UI} / \mathrm{L}, P=0<0,01$ ).

A positividade ao anti-VHC nos doadores de sangue na população de Curitiba no mesmo período em que foi realizado o estudo, foi de $0,65 \%$. A prevalência de infecção pelo VHC encontrada nos 145 pacientes com DM tipo 2 foi estatisticamente superior aos valores obtidos nos doadores de sangue ( $2 \%$ vs. $0,65 \% ; P=0,04$; OR 3,2; 95\% IC $=1,00-10,21$ ). Cabe ressaltar que com esta amostra obteve-se poder de teste de $75 \%$, considerando um erro do tipo I de $5 \%$.

\section{DISCUSSÃO}

O presente estudo encontrou prevalência aumentada de infecção pelo VHC em pacientes com DM tipo 2, se comparada à população adulta saudável de Curitiba, representada pelos doadores de sangue. A prevalência de anti-VHC neste grupo está de acordo com os dados de inquérito nacional conduzido pela Sociedade Brasileira de Hepatologia e recentemente publicado ${ }^{(7)}$, no qual se encontrou prevalência de anticorpos para hepatite $\mathrm{C}$ em 0,65\% de 377066 doadores de sangue testados na região sul do país.

$\mathrm{O}$ achado de prevalência aumentada da infecção pelo VHC em diabéticos tipo 2 em nosso meio corrobora as observações de outros pesquisadores que encontraram índices variando de 3,09\% 
até $28 \%$, sendo que os maiores índices são encontrados quando se analisam particularmente os diabéticos com elevação das aminotransferases ${ }^{(5,14,17,18,19)}$. Neste estudo os pacientes com DM tipo 2 apresentaram taxas mais elevadas de ALT que os diabéticos tipo $1(P<0,01)$. Diversos fatores podem contribuir para a elevação das taxas de ALT em pacientes com DM tipo 2, independentemente da presença de VHC, tais como obesidade e esteatose hepática, condições que podem acompanhar os quadros de resistência insulínica.

Ao se postular sobre os fatores capazes de explicar a associação entre infecção pelo VHC e DM, uma das hipóteses aventadas é que os pacientes com DM são freqüentemente expostos a situações de risco para contrair hepatite $\mathrm{C}$ durante as hospitalizações. Investigando tal hipótese RUDONI et al. ${ }^{(17)}$ encontraram prevalência de 3,09\% de anti-VHC (+) em 259 diabéticos, não observando diferença na freqüência de exposição a possíveis situações de risco como tipo de tratamento, hospitalizações prévias em unidades de tratamento de diabetes, uso de medidores de glicemia por punção digital entre os pacientes anti-VHC positivos e os negativos. Esses autores sugerem que tais práticas não são relevantes na transmissão nosocomial do VHC nessa população. No presente estudo, embora com amostra reduzida, não se encontrou diferença estatisticamente significante na exposição a fatores de risco entre pacientes diabéticos tipo 2 anti-VHC positivos e negativos.

Outra hipótese mais atraente para elucidar a associação entre VHC e DM seria a possibilidade da infecção pelo VHC atuar como fator adicional na instalação de DM tipo 2 em indivíduos com outros fatores de risco reconhecidos para essa enfermidade. METHA et al. ${ }^{(13)}$ acompanharam prospectivamente 1084 adultos, dos quais 548 desenvolveram DM ao longo de 9 anos de seguimento. Os pacientes foram categorizados como de baixo e alto risco para DM, de acordo com idade e índice de massa corporal. Entre os pacientes de alto risco para DM, os com infecção pelo VHC estavam 11 vezes mais predispostos a desenvolver DM do que aqueles sem a infecção (risco relativo 11,58; 95\% IC: 1,139-96,6). Esses dados preliminares sugerem que a infecção pelo VHC serviria como fator de risco adicional para instalação de DM tipo 2 em pessoas com outros fatores de risco estabelecidos para diabetes. Estudos recentes $^{(3,16,20)}$ têm documentado a ocorrência de resistência insulínica em portadores do VHC, mesmo na ausência de doença hepática avançada. A instalação de resistência insulínica poderia ser elo de ligação entre a infecção viral e o desenvolvimento de DM tipo 2 em indivíduos com outros fatores de risco presentes. $\mathrm{O}$ mecanismo pelo qual a infecção pelo $\mathrm{VHC}$ induz à resistência insulínica tem sido alvo de intensa investigação. Algumas das hipóteses aventadas incluem a ação de citocinas, que induzem à resistência insulínica como a interleucina- 6 e fator de necrose tumoral, que habitualmente se elevam na presença de inflamação desencadeada pelo VHC e, mais recentemente, a descoberta que a infecção pelo VHC induz à expressão dos genes de uma proteína que desempenha papel central na via de sinalização da insulina ${ }^{(4,10,21)}$.

Este estudo apresenta limitações visto que aborda apenas um aspecto da possível associação entre VHC e DM, além de trabalhar com população de tamanho limitado. Entretanto, os achados positivos sinalizam como estímulo para que se estude mais profundamente a inter-relação entre a infecção pelo VHC e DM na nossa população. Considerando que além do presente estudo, vários outros autores têm observado prevalência aumentada de infecção pelo VHC em diabéticos tipo 2, tal achado deve ser lembrado na avaliação dessa população, particularmente na presença de alteração das enzimas hepáticas. Por outro lado, os dados recentes que associam a infecção pelo VHC a maior risco de resistência insulínica alertam para importância de controle de fatores de risco sabidamente conhecidos para o DM em portadores do VHC.

Parolin MB, Réa R, Vargas RM, Almeida ACR, Baldanzi GR, Lopes RW. Prevalence of hepatitis C infection in patients with type 2 diabetes mellitus. Arq Gastroenterol 2006;43(2):77-80.

ABSTRACT - Background - Recently, a possible epidemiological association between hepatitis $\mathrm{C}$ virus infection and diabetes mellitus has been suggested and a higher prevalence of HCV antibodies has been found among type 2 diabetic when compared with normal controls. Aim - To evaluate the prevalence of hepatitis C infection in diabetic patients in Curitiba, PR, Brazil. Patients and Methods - A total of 145 type 2 and 104 type 1 diabetic patients attending the outpatient diabetic unit of an university hospital were consecutively tested for anti-HCV, using a fourth-generation enzyme-linked immunosorbent assay (ELISA). The control group was constituted by 16,720 volunteer blood donors attending the blood bank of the same hospital during the period of the study. Diabetic patients were also evaluated for clinical, biochemical (aminotransferase levels) and demographic variables and previous exposure to risk factors for hepatitis C infection. Results - A higher prevalence of hepatitis $\mathrm{C}$ infection was observed in type 2 diabetic patients in comparison with blood donors. Although anti-HCV prevalence in type 2 diabetic patients was higher than found in type 1, it did not reach statistical significance. Both diabetic groups were predominantly female, and as expected, type 2 diabetic were older than type 1. Race distribution, duration of the disease, and previous exposure to hepatitis $\mathrm{C}$ risk factors were similar in both groups, but type 2 diabetic subjects had higher median levels of alanine aminotransferase than type 1. Conclusions - A higher prevalence of hepatitis C infection was detected in type 2 diabetic patients in comparison with blood donors in our region, in accordance with study data from different populations. If all type 2 diabetic patients should undergo regular screening for hepatitis $\mathrm{C}$ infection remains a question.

HEADINGS - Hepatitis C. Diabetes mellitus, type II. 


\section{REFERÊNCIAS BIBLIOGRÁFICAS}

1. Allison MED, Wreighitt T, Palmer CR, Alexander GL. Evidence for a link between hepatitis $\mathrm{C}$ infection and diabetes mellitus in a cirrhotic population. J Hepatol. 1994;21:1135-9.

2. Caronia S, Taylor K, Pagliaro L, Carr C, Palazzo U, Petrik J, O'Rahilly S, Shore S, Tom BDM, Alexander GJM. Further evidence for an association between non-insulin dependent diabetes mellitus and chronic hepatitis $\mathrm{C}$ virus infection. Hepatology. 1999;30:1059-63.

3. Delgado-Borrego A, Casson D, Schoenfeld D, Somsouk M, Terella A, Jordan SH, Bhan A, Baid S, Cosimi AB, Pascual M, Chung R. Hepatitis C virus is independently associated with increased insulin resistance after liver transplantation. Transplantation 2004;77:703-10.

4. Fukui M, Kitagawa Y, Nakamura N, Yoshikawa T. Response to Öncül. Insulin sensitivity in patients with chronic hepatitis $\mathrm{C}$ virus infection [letter]. Diabetes Care. 2002;25:1900-1.

5. Gray H, Wreghitt T, Stratton IM, Alexander GJ, Turner RC, O'Rahilly S. High prevalence of hepatitis $\mathrm{C}$ infection in Afro-Caribbean patients with type 2 diabetes and abnormal liver function tests. Diabet Med. 1995;12:244-9.

6. Grimbert S, Valnesi P, Levy-Marchal C, Perret G, Richardet JP, Raffoux C, Trinchet JC, Beaugrand M. High prevalence of diabetes mellitus in patients with chronic hepatitis C. A case-control study. Gastroenterol Clin Biol. 1996;20:544-8.

7. Grupo de Estudo da Sociedade Brasileira de Hepatologia. Epidemiologia da infecção pelo vírus da hepatite C no Brasil. GED Gastroenterol Endosc Dig. 1999;18:S3-S8.

8. Hoofnagle JH. Course and outcome of hepatitis C. Hepatology. 2002;36:S21-S9.

9. Knobler H, Schihmanter R, Zifroni A, Fenakel G, Schatter A. Increased risk of type 2 diabetes in cirrhotic patients with chronic hepatitis $\mathrm{C}$ virus infection. Mayo Clin Proc 2000;75:355-9.

10. Malaguarnera M, Di Fazio I, Romeo MA, Restuccia S, Laurino A, Trovato BA Elevation of interleukin 6 levels in patients with chronic hepatitis due to hepatitis $\mathrm{C}$ virus. J Gastroenterol. 1997;32:211-5.

11. Mason AL, Lau JYN, Hoang N, Qian K, Alexander GLM, Xu L, Guo L, Jacob S, Regenstein FG, Zimmerman RP, Everhart JE, Wasserfall C, MacLaren NK, Perrillo R. Association of diabetes mellitus and chronic hepatitis C virus infection. Hepatology 1999;29:328-33.
12. Metha SH, Strathdee SA, Thomas DL. Association between hepatitis C infection and diabetes mellitus. Epidemiol Rev. 2001;23:302-12.

13. Metha SH, Brancatti FL, Strathdee SA, Pankow JS, Netski D, Coresh J, Szklo M, Thomas DL. Hepatitis $\mathrm{C}$ virus infection and incident type 2 diabetes. Hepatology. 2003;38:50-66

14. Okan V, Araz M, Aktaran S, Karsligil T, Meram I, Bayraktaroglu Z, Demirci F. Increased frequency of $\mathrm{HCV}$ but not $\mathrm{HBV}$ infection in type 2 diabetic patients in Turkey. Int J Clin Pract. 2002;56:175-7.

15. Ozylikan E, Erbas T, Simsek H, Telatar F, Kayhan B, Telatar H. Increased prevalence of hepatitis $\mathrm{C}$ virus antibodies in patients with diabetes mellitus [letter]. J Intern Med. 1994;235:283-5.

16. Petit J-M, Bour J-B, Galland-Jas C, MInello A, Verges B, Guiguet M, Brun J-M, Hilton P. Risk factors for diabetes mellitus and early insulin resistance in chronic hepatitis C. J Hepatol. 2001;35:279-83.

17. Rudoni S, Petit JM, Bour JB, Aho LS, Castaneda A, Vaillant G, Verrges B, Brun JM $\mathrm{HCV}$ infection and diabetes mellitus: influence of the use of finger stick devices on nosocomial transmission. Diabetes Metab. 1999;25:502-5.

18. Sangiorgio L, Attardo T, Gangemi R, Rubino C, Barone M, Lunetta M. Increased frequency of $\mathrm{HCV}$ and $\mathrm{HBV}$ infection in type 2 diabetic patients. Diabetes Res Clin Pract. 2000;48:147-51.

19. Simo R, Hernadez C, Genesca J, Jardi R, Mesa J. High prevalence of hepatitis C infection in diabetic patients. Diabetes Care. 1996;19:998-1000.

20. Sougleri M, Labropoulou-Karatza C, Paraskevopoulou P, Fragopanagou H, Alexandrides T. Chronic hepatitis $\mathrm{C}$ virus infection cirrhosis induces insulin resistance in patients with -thalassaemia major. Eur J Gastroenterol Hepatol. 2001;13:1195-9.

21. Su AI, Pezacki JP, Wodicka L, Brideau AD, Superkova L, Thimme R, Wieland S, Bukh J, Purcell RH, Schultz PG, Chisari FV. Genomic analysis of the host response to hepatitis C virus infection. Proc Natl Acad Sci USA. 2002;99:15669-74.

Recebido em 27/6/2005 Aprovado em 14/10/2005. 